\title{
FUNCTIONS OF UNIFORMLY BOUNDED CHARACTERISTIC
}

\author{
SHINJI YAMASHITA
}

\section{Introduction}

We shall introduce a new notion of functions of uniformly bounded characteristic in the disk in terms of the Shimizu-Ahlfors characteristic function.

Let $f$ be a function meromorphic in the disk $D=\{|z|<1\}$ in the complex plane $\boldsymbol{C}=\{|z|<\infty\}$. Let $f^{\#}=\left|f^{\prime}\right| /\left(1+|f|^{2}\right), 0<r<1$, and $z=x+i y$. Set

$$
S(r, f)=(1 / \pi) \iint_{|z|<r} f^{\#}(z)^{2} d x d y .
$$

The Shimizu-Ahlfors characteristic function of $f$,

$$
T(r, f)=\int_{0}^{r} t^{-1} S(t, f) d t,
$$

is a non-decreasing function of $r, 0<r<1$, so that

exists.

$$
T(1, f)=\lim _{r \rightarrow 1} T(r, f) \leqq \infty,
$$

Let BC be the family of $f$ meromorphic in $D$ with $T(1, f)<\infty$. Then, $g$ meromorphic in $D$ is of bounded (Nevanlinna) characteristic in $D$ if and only if $g \in \mathrm{BC}$. Letting $w \in D$ as a parameter we set

$$
\varphi_{w}(z)=(z+w) /(1+\bar{w} z), \quad z \in D .
$$

The inverse map of $\varphi_{w}$ is then $\varphi_{-w}$. We set $f_{w}(z)=f\left(\varphi_{w}(z)\right), z \in D$. If $f \in \mathrm{BC}$, then $f_{w} \in \mathrm{BC}$ for all $w \in D$.

Definition. A meromorphic function $f$ in $D$ is said to be of uniformly bounded characteristic in $D$ if and only if

$$
\sup _{w \in D} T\left(1, f_{w}\right)<\infty .
$$

Denote by UBC the family of meromorphic functions in $D$ of uniformly bounded characteristic in $D$. By $\mathrm{UBC}_{0}$ we mean the family of functions $f$ meromorphic in $D$ 
such that

$$
\lim _{|w| \rightarrow 1} T\left(1, f_{w}\right)=0 .
$$

Then $\mathrm{UBC} \subset \mathrm{BC}$. However, the inclusion formula $\mathrm{UBC}_{0} \subset \mathrm{UBC}$ is never obvious and needs a proof (Lemma 2.1.).

In Section 2 we propose a criterion (Theorem 2.2) for a meromorphic $f$ to belong to $\mathrm{UBC}$ or $\mathrm{UBC}_{0}$ in terms of the Green function of $D$.

In Section 3 we show that $\mathrm{UBC}$ is a subfamily of the family $\mathrm{N}$ of meromorphic functions normal in $D$ in the sense of O. Lehto and K. I. Virtanen [5]; an analogue: $\mathrm{UBC}_{0} \subset \mathrm{N}_{0}$, is also considered (Theorem 3.1). Use is made of J. Dufresnoy's lemma $\left[1\right.$, p. 218], from which a criterion for $f$ to be of $N$ or of $N_{0}$ is obtained in terms of the spherical areas of the Riemannian images of the non-Euclidean disks (Lemma 3.2). We believe that this criterion itself is novel.

In Section 4 we consider Blaschke products

$$
\begin{gathered}
b(z)=z^{k} \Pi \frac{\left|a_{n}\right|}{a_{n}} \frac{a_{n}-z}{1-\bar{a}_{n} z} \\
\left(k \geqq 0 \text { integer; } \sum\left(1-\left|a_{n}\right|\right)<\infty\right) .
\end{gathered}
$$

If $f \in \mathrm{UBC}$ is not identically zero, then $f$, as a member of $\mathrm{BC}$, has the decomposition $b_{1} g / b_{2}$, where $g \in \mathrm{BC}$ is pole- and zero-free, and $b_{1}$ and $b_{2}$ are Blaschke products without common zeros. We observe that $g \in \mathrm{UBC}$. One of the essential differences of $\mathrm{UBC}$ from $\mathrm{BC}$ is that $\mathrm{UBC}$ is not closed for summation and multiplication. This is a consequence of Theorem 4.2. For the proof, Blaschke products play fundamental roles.

In Section 5 holomorphic functions $f$ in $D$ are considered. A criterion for $f \in \mathrm{UBC}$ or $f \in \mathrm{UBC}_{0}$ is obtained in terms of the harmonic majorants (Theorem 5.1). In Theorem 5.2 we claim that if the image $f(D)$ is contained in a domain in $C$ of a certain type, then $f \in \mathrm{UBC}$.

If $f$ is holomorphic and bounded in $D$, then $f \in \mathrm{UBC}$. In Section 6 we show that if a meromorphic $f$ satisfies the condition

$$
\iint_{D}^{\#}(z)^{2} d x d y<\infty
$$

then $f \in \mathrm{UBC}$. Thus, if $f$ is "bounded" in a natural sense, then $f \in \mathrm{UBC}$.

In the final section, Section 7, we consider BMOA and VMOA functions. These are, roughly speaking, holomorphic functions in $D$ whose boundary values are of bounded or vanishing mean oscillation on the circle $\{|z|=1\}$ in the sense of $\mathrm{F}$. John and L. Nirenberg [4] or of D. Sarason [7], respectively. The main result is that $\mathrm{BMOA} \subset \mathrm{UBC}$ and $\mathrm{VMOA} \subset \mathrm{UBC}_{0}$.

To extend the notion of $\mathrm{UBC}$ and $\mathrm{UBC}_{0}$ (as well as BMOA and VMOA) to Riemann surfaces $R$ is possible. Some arguments in $D$ are also available on $R$. We hope we can publish a systematic study of $\mathrm{UBC}$ and $\mathrm{UBC}_{0}$ on $R$ in the near future. 


\section{Criteria}

First we show, as was promised in Section 1 , that $\mathrm{UBC}_{0} \subset \mathrm{UBC}$; for the proof, use is made of

Theorem 2.1. If $f \in \mathrm{BC}$, then for each $\varrho, 0<\varrho<1$,

$$
\sup _{|w|<\varrho} T\left(1, f_{w}\right)<\infty \text {. }
$$

Proof. Set for $w \in D$ and for $\lambda, 0<\lambda<1$,

$$
\Delta(w, \lambda)=\{z \in D ;|w-z| /|1-\bar{w} z|<\lambda\}
$$

this is the non-Euclidean disk of the non-Euclidean center $w$ and the non-Euclidean radius $(1 / 2) \log [(1+\lambda) /(1-\lambda)]$. The change of variable $\zeta=\xi+i \eta=\varphi_{w}(z)$ then yields that

$$
S\left(\lambda, f_{w}\right)=(1 / \pi) \iint_{|z|<\lambda} f_{w}^{\#}(z)^{2} d x d y=(1 / \pi) \iint_{\Delta(w, \lambda)} f^{\#}(\zeta)^{2} d \xi d \eta
$$

hereafter, $\left(f_{w}\right)^{\#}=f_{w}^{\#}$ and $\left(\varphi_{w}\right)^{\prime}=\varphi_{w}^{\prime}$ for short.

Fix $\varrho, 0<\varrho<1$, and then let $w$ satisfy $|w|<\varrho$. For $r_{0} \equiv 1 / 2<r<1$, we shall estimate upwards the characteristic function

$$
T\left(r, f_{w}\right)=T\left(r_{0}, f_{w}\right)+\int_{r_{0}}^{r} t^{-1} S\left(t, f_{w}\right) d t \equiv \alpha+\beta
$$

by a constant independent of $r$ and $w$.

For the $\alpha$-part we note that

$$
|z|<r_{0} \Rightarrow\left|\varphi_{w}(z)\right| \leqq(|w|+|z|) /(1+|z w|)<R_{0} \equiv\left(r_{0}+\varrho\right) /\left(1+r_{0} \varrho\right) .
$$

Then, for $|z|<r_{0}$,

$$
f_{w}^{\#}(z)=f^{\#}\left(\varphi_{w}(z)\right)\left|\varphi_{w}^{\prime}(z)\right| \leqq\left[\max _{|\zeta| \leqq R_{0}} f^{\#}(\zeta)\right]\left(1-\varrho r_{0}\right)^{-2} \equiv K<\infty
$$

by the continuity of $f^{\#}$. Consequently,

$$
f_{w}^{\#}(z) \leqq K \text { for }|z|<t<r_{0},
$$

so that the inequality $S\left(t, f_{w}\right) \leqq K^{2} t^{2}$ yields

$$
\alpha \leqq K^{2} / 8 .
$$

To estimate $\beta$ we notice that, for $0<t<1$,

$$
\Delta(w, t) \subset\{|z|<u\}, \quad u \equiv(t+\varrho) /(1+\varrho t) .
$$

By (2.1), together with $R \equiv(r+\varrho) /(1+r \varrho)>R_{0}$, we obtain

$$
\beta \leqq \int_{r_{0}}^{r} t^{-1} S(u, f) d t=\int_{R_{0}}^{R} C(u, \varrho) u^{-1} S(u, f) d u,
$$


where

$$
C(u, \varrho)=\frac{u\left(1-\varrho^{2}\right)}{(u-\varrho)(1-\varrho u)} \leqq 2 /\left(R_{0}-\varrho\right)
$$

because $\varrho<R_{0}<u<1$ for $r_{0}<t<r$. Therefore

$$
\beta \leqq 2 T(R, f) /\left(R_{0}-\varrho\right) \leqq 2 T(1, f) /\left(R_{0}-\varrho\right),
$$

which, together with (2.2), completes the proof.

Lemma 2.1. $\mathrm{UBC}_{0} \subset \mathrm{UBC}$.

Proof. For $f \in \mathrm{UBC}_{0}$ there exists $\delta, 0<\delta<1$, such that $T\left(1, f_{w}\right)<1$ in $\{\delta<|w|<1\}$. Then $f \in \mathrm{BC}$ because $f$ is the composed function $f=f_{\varrho} \circ \varphi_{-\varrho}$ for $\varrho=(1+\delta) / 2$ with $f_{\ell} \in \mathrm{BC}$. It now follows from Theorem 2.1 that

whence

$$
K \equiv \sup _{|w|<\varrho} T\left(1, f_{w}\right)<\infty,
$$

$$
\sup _{w \in D} T\left(1, f_{w}\right) \leqq K+1 .
$$

Remark. Theorem 2.1 also yields:

For $f$ meromorphic in $D$ to be of UBC it is necessary and sufficient that

$$
\limsup _{|w| \rightarrow 1} T\left(1, f_{w}\right)<\infty \text {. }
$$

The Green function of $D$ with pole at $w \in D$ is given by

$$
G(z, w)=\log |(1-\bar{w} z) /(z-w)|=-\log \left|\varphi_{-w}(z)\right|, \quad z \in D .
$$

We now propose the main result in the present section.

Theorem 2.2. Let $f$ be meromorphic in $D$. Then the following propositions hold.

(I) $f \in \mathrm{UBC}$ if and only if

$$
\sup _{w \in D} \iint_{D} f^{\#}(z)^{2} G(z, w) d x d y<\infty .
$$

(II) $f \in \mathrm{UBC}_{0}$ if and only if

$$
\lim _{\{w \mid \rightarrow 1} \iint_{D} f^{\#}(z)^{2} G(z, w) d x d y=0 .
$$

For the proof we need

Lemma 2.2. For $f$ meromorphic in $D$ and for $0<r \leqq 1$ we have

$$
T(r, f)=(1 / \pi) \iint_{|z|} f_{r}^{\#}(z)^{2} \log (r /|z|) d x d y .
$$

Proof. For $0<r<1$, we let $X_{r}$ be the characteristic function of the disk $\{|z|<r\}$, namely, $X_{\boldsymbol{r}}(z)=1$ for $|z|<r, \quad X_{r}(z)=0$ for $r \leqq|z|<1$. 
It suffices to prove (2.5) for $0<r<1$. For, if (2.5) is true for $0<r<1$, then

$$
T(r, f)=(1 / \pi) \iint_{D} f^{\#}(z)^{2} X_{r}(z) \log (r /|z|) d x d y .
$$

Since $0 \leqq X_{r}(z) \log (r /|z|) / \log (1 /|z|)$ as $r \rightarrow 1$ at each $z \in D$, (2.5) for $r=1$ follows.

Now, for $0<r<1$,

$$
\begin{array}{rlrlrl}
\int_{0}^{r} t^{-1} X_{t}(z) d t & =\log (r /|z|) & & \text { if } & & |z|<r, \\
& =0 & & \text { if } & r \leqq|z|<1,
\end{array}
$$

so that $(2.5)$ is a consequence of

$$
T(r, f)=(1 / \pi) \iint_{D} f^{\#}(z)^{2}\left[\int_{0}^{r} t^{-1} X_{t}(z) d t\right] d x d y .
$$

Proof of Theorem 2.2. Since $f_{w}^{\#}=\left(f^{\#} \circ \varphi_{w}\right)\left|\varphi_{w}^{\prime}\right|$, it follows from Lemma 2.2, together with the change of variable $\zeta=\varphi_{w}(z)$, that

$$
T\left(1, f_{w}\right)=(1 / \pi) \iint_{D} f^{\#}(\zeta)^{2} \log \left(1 /\left|\varphi_{-w}(\zeta)\right|\right) d \xi d \eta .
$$

This completes the proof of Theorem 2.2.

Remark. For $f \in \mathrm{BC}$, the function $T\left(1, f_{w}\right)$ of $w \in D$ is well defined. The identity (2.6) shows that $T\left(1, f_{w}\right)$ is lower semicontinuous with respect to $w \in D$. Actually, $T\left(1, f_{w}\right)$ is a Green's potential in $D$ of the measure in the differential form

$$
(1 / \pi) f^{\#}(\zeta)^{2} d \xi d \eta \text {. }
$$

\section{Normal meromorphic functions}

Let $\mathrm{N}$ be the family of meromorphic functions $f$ in $D$ such that

$$
\sup _{z \in D}\left(1-|z|^{2}\right) f^{\#}(z)<\infty,
$$

and let $\mathrm{N}_{0}$ be the family of meromorphic functions $f$ in $D$ such that

$$
\lim _{|z| \rightarrow 1}\left(1-|z|^{2}\right) f^{*}(z)=0 \text {. }
$$

Each $f \in \mathrm{N}$ is normal in $D$ in the sense of Lehto and Virtanen [5], and vice versa. By the continuity of $f^{*}$, the inclusion formula $\mathrm{N}_{0} \subset \mathrm{N}$ is easily established.

Theorem 3.1. The following inclusion formulae hold:

$$
\mathrm{UBC} \subset \mathrm{N} \text { and } \mathrm{UBC}_{0} \subset \mathrm{N}_{0} \text {; }
$$

both are shown to be sharp.

We begin with Dufresnoy's result. 
Lemma 3.1 [1, Lemma, p. 218] (See [3, Theorem 6.1, p. 152].). Suppose that $f$ is meromorphic in $D$ and that there exists $r, 0<r<1$, such that $S(r, f)<1$. Then

$$
f^{\#}(0)^{2} \leqq S(r, f) r^{-2}[1-S(r, f)]^{-1} .
$$

Note that our Riemann sphere is of radius $1 / 2$, touching $C$ from above at 0 , while Dufresnoy considered the sphere of radius 1 bisected by $\boldsymbol{C}$.

Lemma 3.2. Let $f$ be meromorphic in D. Then the following propositions hold. (I) $f \in \mathrm{N}$ if and only if there exists $r, 0<r<1$, such that

$$
\sup _{w \in D} S\left(r, f_{w}\right)=(1 / \pi) \sup _{w \in D} \iint_{\Delta(w, r)} f^{\#}(z)^{2} d x d y<1 .
$$

(II) $f \in \mathrm{N}_{0}$ if and only if there exists $r, 0<r<1$, such that

$$
\lim _{|w| \rightarrow 1} S\left(r, f_{w}\right)=\lim _{|w| \rightarrow 1} \iint_{\Delta(w, r)} f^{\#}(z)^{2} d x d y=0 .
$$

In the proof of Theorem 3.1, the "if" parts of (I) and (II) are needed. Lemma 3.2 (I) gives a new criterion for $f$ to be normal in $D$.

There exist a nonnormal holomorphic function $f$ and $r>0$ for which $S\left(r, f_{w}\right)<1$ for each $w \in D$; see [12, Remark, p. 226]. This function $f$ must satisfy

$$
\sup _{w \in D} S\left(r, f_{w}\right)=1 .
$$

Proof of Lemma 3.2. For the proof of (I) we first assume that $f \in \mathrm{N}$ with

$$
\left(1-|z|^{2}\right) f^{\#}(z) \leqq K<\infty \text { for all } z \in D .
$$

Then, for each $w \in D$,

$$
\left(1-|z|^{2}\right) f_{w}^{\#}(z)=\left(1-\left|\varphi_{w}(z)\right|^{2}\right) f^{\#}\left(\varphi_{w}(z)\right) \leqq K, \quad z \in D .
$$

Therefore, for a small $r, 0<r<1$, with $K^{2} r^{2} /\left(1-r^{2}\right)<1$,

$$
\pi S\left(r, f_{w}\right)=\iint_{|z|} f_{<r}^{\#}(z)^{2} d x d y \leqq 2 \pi K^{2} \int_{0}^{r} \varrho\left(1-\varrho^{2}\right)^{-2} d \varrho=\pi K^{2} r^{2} /\left(1-r^{2}\right),
$$

whence (3.1) follows. Conversely, let the supremum in (3.1) be $S$. Then, by Lemma 3.1 , together with $x /(1-x) /$ as $0 \leqq x / 1$,

$$
\left(1-|w|^{2}\right)^{2} f^{\#}(w)^{2}=f_{w}^{\#}(0)^{2} \leqq r^{-2} S(1-S)^{-1}
$$

for all $w \in D$, whence $f \in \mathrm{N}$.

To prove (II) we first suppose that $f \in \mathrm{N}_{0}$. Then, for each $\varepsilon>0$, there exists $\delta, 0<\delta<1$, such that

$$
\delta<|z|<1 \Rightarrow\left(1-|z|^{2}\right) f^{\#}(z)<\varepsilon^{1 / 2} .
$$


Choose $r$ such that $0<r<\delta$ and $r^{2} /\left(1-r^{2}\right)<1$. Then

$$
\delta<(r+\delta) /(1+r \delta)<|w|<1 \Rightarrow \Delta(w, r) \subset\{\delta<|z|<1\}
$$

because

$$
\delta<(|w|-r) /(1-r|w|)<|z| \text { for } \quad z \in \Delta(w, r) .
$$

The formula (2.1), together with (3.3) and (3.4), yields that

$$
\pi S\left(r, f_{w}\right)=\iint_{\Delta(w, r)} f^{\#}(z)^{2} d x d y \leqq \varepsilon \pi r^{2} /\left(1-r^{2}\right) ;
$$

in fact, the non-Euclidean area of $\Delta(w, r)$ is $\pi r^{2} /\left(1-r^{2}\right)$. Therefore,

$$
S\left(r, f_{w}\right)<\varepsilon \text { for }(r+\delta) /(1+r \delta)<|w|<1 .
$$

Conversely, suppose that (3.2) holds. Then, for each $\varepsilon>0$, there exists $\delta, 0<\delta<1$, such that

$$
S\left(r, f_{w}\right)<\varrho \text { for } \delta<|w|<1,
$$

where $0<\varrho<1$ and $\varrho r^{-2}(1-\varrho)^{-1}<\varepsilon / 2$. By Lemma 3.1,

$$
\left(1-|w|^{2}\right)^{2} f^{\#}(w)^{2}=f_{w}^{\#}(0)^{2}<\varepsilon \text { for } \delta<|w|<1,
$$

which completes the proof.

Remark. The condition (3.1) can be replaced by

$$
\limsup _{|w| \rightarrow 1} S\left(r, f_{w}\right)<1 \text {. }
$$

Proof of Theorem 3.1. Suppose that $f \in$ UBC. Then (2.3) of Theorem 2.2 holds; we denote by $A$ the supremum in (2.3). Choose $r, 0<r<1$, such that

$$
A /[\pi \log (1 / r)]<1
$$

Since, for each $w \in D$, the formula (2.1) yields that

$$
A \geqq \iint_{\Delta(w, r)} f^{\#}(z)^{2} G(z, w) d x d y \geqq \pi \log (1 / r) S\left(r, f_{w}\right),
$$

it follows from Lemma 3.2, (I), together with (3.5), that $f \in \mathrm{N}$. Therefore $\mathrm{UBC} \subset \mathrm{N}$. The proof of $\mathrm{UBC}_{0} \subset \mathrm{N}_{0}$ is similar.

To prove the sharpness it suffices to observe the existence of $f \in \mathrm{N}_{0}-\mathrm{BC}$. Then $f \in \mathrm{N}_{0}-\mathrm{UBC}_{0}$ and $f \in \mathrm{N}-\mathrm{UBC}$. Consider the gap series

$$
f(z)=\sum_{k=1}^{\infty} a_{k} z^{n_{k}}, \quad z \in D,
$$

where the sequence $\left\{n_{k}\right\}$ of positive integers satisfies $n_{k+1} / n_{k} \geqq q>1$ for all $k \geqq 1$. Suppose that

$$
\sum_{k=1}^{\infty}\left|a_{k}\right|^{2}=\infty \text { and } \lim _{k \rightarrow \infty}\left|a_{k}\right|=0
$$


Then it is known (see [10, Corollary, p. 34]) that

$$
\lim _{|z| \rightarrow 1}\left(1-|z|^{2}\right)\left|f^{\prime}(z)\right|=0
$$

and $f$ does not have finite radial limit a.e. on $\{|z|=1\}$. Therefore, $f \in \mathrm{N}_{0}$, yet $f \notin \mathrm{BC}$.

\section{Blaschke products}

First of all we prove

Lemma 4.1. Suppose that $f \in \mathrm{UBC}$ and that $g$ is a rational function. Then $g \circ f \in \mathrm{UBC}$.

Proof. There exists $K>0$ such that

$$
g^{\#}(z) \leqq K /\left(1+|z|^{2}\right) \text { for all } z \in C .
$$

Since $(g \circ f)_{w}=g \circ f_{w}$, it follows that

Consequently,

$$
(g \circ f)_{w}^{\#}=\left(g \circ f_{w}\right)^{\#}=\left(g^{\#} \circ f_{w}\right)\left|f_{w}^{\prime}\right| \leqq K f_{w}^{\#} .
$$

$$
T\left(1,(g \circ f)_{w}\right) \leqq K^{2} T\left(1, f_{w}\right),
$$

which shows that $g \circ f \in \mathrm{UBC}$.

As we shall observe later in Theorem 4.2, UBC is not closed for summation and multiplication. The family UBC resembles $\mathrm{N}$ at this point. However, a decisive difference between $\mathrm{UBC}$ and $\mathrm{N}$ is that, each non-zero $f \in \mathrm{UBC}$, as a member of $\mathrm{BC}$, admits the decomposition

$$
f=b_{1} g / b_{2},
$$

where $g \in \mathrm{BC}$ has neither pole nor zero in $D$, and $b_{1}\left(b_{2}\right.$, respectively) is the Blaschke product whose zeros are precisely the zeros (poles, respectively) of $f$, the multiplicity being counted. For simplicity we shall call $b_{2}$ the polar Blaschke product of $f$. If $f$ is pole-free, then $b_{2} \equiv 1$.

We shall show that $g$ of (4.1) is a member of UBC if $f \in \mathrm{UBC}$ as a corollary of

Theorem 4.1. Let $f \in \mathrm{UBC}$, and let $b$ be the polar Blaschke product of $f$. Then $b f \in \mathrm{UBC}$.

For the proof of Theorem 4.1, we first deduce the formula (4.4) in Lemma 4.2 by making use of a precise description of the first step in the Nevanlinna theory. The adjective "precise" in the preceding sentence means that there is no Landau's notation $O(1)$.

Let

$$
I(r, f)=(1 / 4 \pi) \int_{0}^{2 \pi} \log \left(1+\left|f\left(r e^{i t}\right)\right|^{2}\right) d t,
$$


and let $n(r, f)\left(n^{*}(r, f)\right)$ be the number of the poles of $f$ in the disk $\{|z|<r\}$ (on the circle $\{|z|=r\}$ ), the multiplicity being counted, $0<r<1$. Delete from $\{|z|<r\}$ the closed disks, with poles on the closed disk $\{|z| \leqq r\}$ as centers, and with common small radii $\varepsilon>0$, apply the Green formula to $\log \left(1+|f|^{2}\right)$ in the resulting domain, and, finally, let $\varepsilon \rightarrow 0$. Then, for $0<r<1$, the identity $\Delta \log \left(1+|f|^{2}\right)=4 f^{* 2}$ (except for poles of $f$ ) yields

$$
r(d / d r) I(r, f)=S(r, f)-n(r, f)-(1 / 2) n^{*}(r, f) .
$$

Arrange $r>0$ with $n^{*}(r, f) \neq 0$ as

$$
0<r_{0}<\ldots<r_{j}<r_{j+1}<\ldots<1 .
$$

For each $R, r_{0} \leqq R<1$, there is a $j$ such that $r_{j} \leqq R<r_{j+1}$. Divide both sides of (4.2) by $r$, and integrate from $\varepsilon, 0<\varepsilon<r_{0}$, to $R$, to obtain

$$
I(R, f)-I(\varepsilon, f)=\int_{\varepsilon}^{R} r^{-1} S(r, f) d r-\int_{\varepsilon}^{R} r^{-1} n(r, f) d r
$$

where

$$
\int_{\varepsilon}^{R}=\int_{\varepsilon}^{r_{0}}+\left(\sum_{p=1}^{j} \int_{r_{p-1}}^{r_{p}}\right)+\int_{r_{j}}^{R}
$$

Lemma 4.2. Let $b$ be the polar Blaschke product of $f \in \mathrm{BC}$. Then,

$$
T(1, f)=I(1, f)-(1 / 2) \log \left[|b(0)|^{2}+\lim _{z \rightarrow 0}|b(z) f(z)|^{2}\right],
$$

where

$$
I(1, f)=\lim _{r \rightarrow 1} I(r, f) .
$$

Proof. Suppose that 0 is a pole of order $k \geqq 0$. Then

$$
\int_{\varepsilon}^{r_{0}} r^{-1} n(r, f) d r=k\left(\log r_{0}-\log \varepsilon\right)
$$

and, in case $k=0$,

$$
I(\varepsilon, f) \rightarrow(1 / 2) \log \left(1+|f(0)|^{2}\right),
$$

as $\varepsilon \rightarrow 0$, while in case $k>0$,

as $\varepsilon \rightarrow 0$, where

$$
I(\varepsilon, f) \sim-k \log \varepsilon+\log A
$$

$$
A=\lim _{z \rightarrow 0}\left|z^{k} f(z)\right| .
$$

Therefore, $\varepsilon \rightarrow 0$, and then $R \rightarrow 1$ in (4.3) yield

$$
T(1, f)=I(1, f)-(1 / 2) \log \left(1+|f(0)|^{2}\right)-\log |b(0)|
$$


if $k=0$, while if $k>0$, then

$$
\begin{aligned}
T(1, f) & =I(1, f)-\log A-\log \left[\lim _{z \rightarrow 0}\left|z^{-k} b(z)\right|\right] \\
& =I(1, f)-\log \left[\lim _{z \rightarrow 0}|b(z) f(z)|\right],
\end{aligned}
$$

which completes the proof.

As an immediate consequence of (4.4) in Lemma 4.2 we obtain

Lemma 4.3. If $f$ is holomorphic and bounded, $|f| \leqq K$, in $D$, then

$$
T\left(1, f_{w}\right) \leqq I\left(1, f_{w}\right) \leqq(1 / 2) \log \left(1+K^{2}\right) \text { for all } w \in D .
$$

Therefore $f \in \mathrm{UBC}$.

Lemma 4.4. Let $b$ be the polar Blaschke product of $f \in \mathrm{BC}$. Then for each constant $\alpha,|\alpha|=1$,

$$
T(1, \alpha b f) \leqq T(1, f)+(1 / 2) \log 2 .
$$

Proof. By (4.4) in Lemma 4.2, applied to $f$ with $g=b f$, we obtain

$$
T(1, f)=I(1, f)-(1 / 2) \log \left(|b(0)|^{2}+|g(0)|\right)^{2},
$$

and it is apparent that $(\alpha g)^{\#}=g^{\#}$. Therefore,

$$
\begin{gathered}
T(1, \alpha b f)=T(1, g)=I(1, g)-(1 / 2) \log \left(1+|g(0)|^{2}\right) \\
\leqq I(1, b)+I(1, f)-(1 / 2) \log \left(1+|g(0)|^{2}\right) \leqq(1 / 2) \log 2+T(1, f)+(1 / 2) \log A,
\end{gathered}
$$

where

$$
A=\left(|b(0)|^{2}+|g(0)|^{2}\right) /\left(1+|g(0)|^{2}\right) \leqq 1 .
$$

We thus obtain (4.5).

Proof of Theorem 4.1. Let $b^{w}$ be the polar Blaschke product of $f_{w}$. Then $\left|b^{w}\right|=$ $\left|b_{w}\right|$ in $D$. Actually, defining

$$
\psi(z, a)=|z-a| /|1-\bar{a} z|, \quad z \in D,
$$

for $a \in D$, one obtains

$$
\psi\left(z, \varphi_{-w}(a)\right)=\psi\left(\varphi_{w}(z), a\right)
$$

Since $a \in D$ is a pole of order $k \geqq 1$ of $f$ if and only if $\varphi_{-w}(a)$ is a pole of order $k \geqq 1$ of $f_{w}$, it follows from the expression

that

$$
|b(z)|=\prod_{j=1}^{\infty} \psi\left(z, a_{j}\right)
$$

for all $z \in D$.

$$
\left|b^{w}(z)\right|=\prod_{j=1}^{\infty} \psi\left(z, \varphi_{-w}\left(a_{j}\right)\right)=\left|b \circ \varphi_{w}(z)\right|
$$


Now, there is a constant $\alpha,|\alpha|=1$, such that $b_{w}=\alpha b^{w}$. Set $g=b f$. Then $g_{w}=b_{w} f_{w}=\alpha b^{w} f_{w}$. It follows from (4.5) in Lemma 4.4, applied to $f_{w}$, that

$$
T\left(1, g_{w}\right) \leqq T\left(1, f_{w}\right)+(1 / 2) \log 2 \text { for all } w \in D .
$$

Consequently, $g \in \mathrm{UBC}$.

Corollary 4.1. If $f \in \mathrm{UBC}$ with (4.1), then $g \in \mathrm{UBC}$. The converse is false.

Proof. By Theorem 4.1, $b_{1} g=b_{2} f \in U$ UC. By Lemma 4.1,

$$
h \equiv 1 /\left(b_{1} g\right)=(1 / g) / b_{1} \in \mathrm{UBC} \text {. }
$$

Again, by Theorem $4.1,1 / g=b_{1} h \in \mathrm{UBC}$, whence, by Lemma 4.1 once more, $g \in \mathrm{UBC}$. To prove that the converse is false we remember that there exist Blaschke products $b_{1}$ and $b_{2}$ with no common zero in $D$ such that the quotient $b_{1} / b_{2}$ is not normal in $D$; see, for example, [11] and [13]. Therefore, $g \equiv 1 \in \mathrm{UBC}$, yet $f \equiv b_{1} g / b_{2} \notin \mathrm{UBC}$ because $f \notin N$.

Finally in this section we prove

Theorem 4.2.

(I) There exist $f \in \mathrm{UBC}$ and $g \in \mathrm{UBC}$ such that $f g \notin \mathrm{N}$.

(II) There exist $f \in \mathrm{UBC}$ and $g \in \mathrm{UBC}$ such that $f+g \notin \mathrm{N}$.

Combined with the inclusion formula $\mathrm{UBC} \subset \mathrm{N}$, Theorem 4.2 asserts that $\mathrm{UBC}$ is not closed for the product and the sum.

Lemma 4.5. Let $f \in \mathrm{UBC}$, and let $g$ be a holomorphic function bounded from below and above in $D$ :

Then $f g \in \mathrm{UBC}$.

$$
0<m \leqq|g| \leqq M<\infty .
$$

Proof. By Lemma 4.3, $g \in$ UBC. Set

Then,

$$
K=\left(1+M^{2}\right) / \min \left(1, m^{2}\right) \text {. }
$$

whence

$$
1+|f g|^{2} \geqq K^{-1}\left(1+|f|^{2}\right)\left(1+|g|^{2}\right)
$$

$$
(f g)^{\# 2} \leqq \frac{\left|f^{\prime} g\right|^{2}+2\left|f f^{\prime} g g^{\prime}\right|+\left|f g^{\prime}\right|^{2}}{K^{-2}\left(1+|f|^{2}\right)^{2}\left(1+|g|^{2}\right)^{2}} \leqq K^{2}\left(f^{\# 2}+2 f^{\#} g^{\#}+g^{\# 2}\right) .
$$

On the other hand, the Cauchy inequality, together with (2.1), yields

$$
\left[\iint_{\Delta(w, r)} f^{\#}(z) g^{\#}(z) d x d y\right]^{2} \leqq \pi^{2} S\left(r, f_{w}\right) S\left(r, g_{w}\right)
$$

for all $w \in D$ and all $r, 0<r<1$. Consequently, by $(2.1)$, together with $(4,6)$, we obtain

$$
\begin{gathered}
\pi S\left(r,(f g)_{w}\right) \leqq \pi K^{2}\left\{S\left(r, f_{w}\right)+S\left(r, g_{w}\right)+2\left[S\left(r, f_{w}\right) S\left(r, g_{w}\right)\right]^{1 / 2}\right\} \\
\leqq 2 \pi K^{2}\left[S\left(r, f_{w}\right)+S\left(r, g_{w}\right)\right] .
\end{gathered}
$$


Therefore

whence $f g \in \mathrm{UBC}$.

$$
T\left(1,(f g)_{w}\right) \leqq 2 K^{2}\left[T\left(1, f_{w}\right)+T\left(1, g_{w}\right)\right],
$$

Proof of Theorem 4.2. Again we consider the Blaschke products $b_{1}$ and $b_{2}$ such that $b_{1} / b_{2}$ is not normal. To prove (I), set $f=b_{1}$ and $g=1 / b_{2}$. Then $f \in \mathrm{UBC}$ and $g \in \mathrm{UBC}$, yet $f g \notin \mathrm{N}$. To prove (II) we set $f=2 / b_{2}$ and $g=\left(b_{1}-2\right) / b_{2}$. Then $f \in$ UBC. Since $1<\left|b_{1}-2\right|<3$ and $1 / b_{2} \in \mathrm{UBC}$, it follows from Lemma 4.5 that $g \in \mathrm{UBC}$. However, $f+g=b_{1} / b_{2} \notin \mathrm{N}$.

\section{Harmonic majorant}

Let $u \not \equiv-\infty$ be a subharmonic function in a domain $\mathscr{D} \subset C$. We call $h$ a harmonic majorant of $u$ in $\mathscr{D}$ if $h$ is harmonic and $u \leqq h$ in $\mathscr{D}$. If $u$ has a harmonic majorant in $\mathscr{D}$, then $u$ has the least harmonic majorant $\hat{u}$ in $\mathscr{D}$, that is, $\hat{u}$ is a harmonic majorant of $u$ in $\mathscr{D}$ and $\hat{u} \leqq h$ for each harmonic majorant $h$ of $u$ in $\mathscr{D}$. In the special case $\mathscr{D}=D, \hat{u}$ is given by the limiting function

$$
\hat{u^{\wedge}}(z)=\lim _{r \rightarrow 1}(1 / 2 \pi) \int_{0}^{2 \pi} u\left(r e^{i t}\right) \frac{r^{2}-|z|^{2}}{\left|r e^{i t}-z\right|^{2}} d t, \quad z \in D .
$$

Theorem 5.1. Let $f$ be holomorphic in $D$. Then the following criteria hold for the subharmonic function $F=(1 / 2) \log \left(1+|f|^{2}\right)$ in $D$.

(I) $f \in \mathrm{UBC}$ if and only if

$$
\sup _{w \in D}\left(F^{\wedge}(w)-F(w)\right)<\infty .
$$

(II) $f \in \mathrm{UBC}_{0}$ if and only if

$$
\lim _{|w| \rightarrow 1}\left(F^{\wedge}(w)-F(w)\right)=0 .
$$

Lemma 5.1. Suppose that a subharmonic function $u$ in $D$ has a harmonic majorant in $D$. Then $\left(u \circ \varphi_{w}\right)^{\wedge}=\hat{u} \circ \varphi_{w}$ for each $w \in D$.

Proof. Since $\hat{u} \circ \varphi_{w}$ is a harmonic majorant of $u \circ \varphi_{w}$ for each $w \in D$, it follows that

$$
\left(u \circ \varphi_{w}\right)^{\wedge} \leqq u^{\wedge} \circ \varphi_{w} .
$$

Apply (5.1) to $v=u \circ \varphi_{w}$ and $\varphi_{-w}$ instead of $u$ and $\varphi_{w}$, respectively. Then

whence

$$
\hat{u}=\left(v \circ \varphi_{-w}\right)^{\wedge} \leqq v^{\wedge} \circ \varphi_{-w},
$$

$$
u^{\wedge} \circ \varphi_{w} \leqq v^{\wedge}=\left(u \circ \varphi_{w}\right)^{\wedge} .
$$

Combining this with (5.1) we have the equality. 
Proof of Theorem 5.1. (I) There exists $K>0$ for $f \in \mathrm{UBC}$ such that $K \geqq T\left(1, f_{w}\right)$ for all $w \in D$. On the other hand, by Lemma 5.1,

whence

$$
I\left(1, f_{w}\right)=\left(F \circ \varphi_{w}\right)^{\wedge}(0)=F^{\wedge} \circ \varphi_{w}(0)=F^{\wedge}(w),
$$

$$
K \geqq T\left(1, f_{w}\right)=I\left(1, f_{w}\right)-(1 / 2) \log \left(1+\left|f_{w}(0)\right|^{2}\right)=F^{\wedge}(w)-F(w) \text { for all } w \in D .
$$

The converse is also true, so that (I) is established. The proof of (II) is similar.

Remarks. (a) We may replace $F$ in the UBC criterion (I) by $\log ^{+}|f|=$ $\max (\log |f|, 0)$ because

$$
\log ^{+}|f| \leqq F \leqq \log ^{+}|f|+(1 / 2) \log 2 .
$$

(b) Suppose that $f \in \mathrm{BC}$ is pole-free. Since $F^{\wedge}$ exists and since the identity

$$
T\left(1, f_{w}\right)=F^{\wedge}(w)-F(w), \quad w \in D,
$$

is also true for the present $f$,

$$
F(w)=F^{\wedge}(w)-T\left(1, f_{w}\right), \quad w \in D,
$$

represents the Riesz decomposition of the subharmonic function $F$ which has a harmonic majorant in $D$. The potential $T\left(1, f_{w}\right)$ is continuous in the present case because the same is true of $F$ and $F^{\wedge}$. The problem is that $T\left(1, f_{w}\right)$ is or is not continuous depending on whether $f$ admits poles in $D$. If $T\left(1, f_{w}\right)$ is proved to be continuous in $D$ for each meromorphic $f \in \mathrm{BC}$, then Theorem 2.1 is immediate.

A subdomain $\mathscr{D}$ of $C$ is called a UBC domain if each holomorphic function $f$ in $D$ which assumes only the values in $\mathscr{D}$ is of UBC. We next consider a criterion for a holomorphic $f$ in $D$ to be of UBC.

Theorem 5.2. Suppose that the function $H(z)=(1 / 2) \log \left(1+|z|^{2}\right)$ has a harmonic majorant in $\mathscr{D} \subset C$, and suppose that $H^{\wedge}-H$ is bounded in $\mathscr{D}$. Then $\mathscr{D}$ is a $\mathrm{UBC}$ domain. The converse is true under the condition that the universal covering surface of $\mathscr{D}$ is conformally equivalent to $D$.

Proof. Let $F=(1 / 2) \log \left(1+|f|^{2}\right)$ for a holomorphic $f: D \rightarrow \mathscr{D}$. The first half follows from $F=H \circ f, F^{\wedge} \leqq H^{\wedge} \circ f$ and Theorem 5.1 (I). To prove the converse we let $p$ be the projection of the universal covering surface $\mathscr{D}^{\infty}$ of $\mathscr{D}$ onto $\mathscr{D}$, and let $q$ be a conformal homeomorphism from $D$ onto $\mathscr{D}^{\infty}$. Then $f=p \circ q \in \mathrm{UBC}$. Since $F=$ $(1 / 2) \log \left(1+|f|^{2}\right)$ and $F^{\wedge}$ both are automorphic with respect to the covering transformations, namely, automorphic with respect to a group of conformal homeomorphisms from $D$ onto $D, H^{\wedge}(z)=F^{\wedge}\left(f^{-1}(z)\right)$ is well-defined in $\mathscr{D}$. Consequently,

implies

$$
F^{\wedge}-F \leqq K \text { in } D \text { by Theorem } 5.1 \text { (I) }
$$

$$
H^{\wedge}-H \leqq K \text { in } \mathscr{D} \text {. }
$$




\section{Riemannian image of finite spherical area}

In this short section we prove

Theorem 6.1. Suppose that a meromorphic function $f$ in $D$ satisfies

$$
\iint_{D} f^{\#}(z)^{2} d x d y<\infty .
$$

Then $f \in \mathrm{UBC} \cap \mathrm{N}_{0}$.

See the remark at the end of the next section.

Proof of Theorem 6.1. For the proof of $f \in \mathrm{N}_{0}$ we set

$$
A=\iint_{D} f^{\#}(z)^{2} d x d y,
$$

and we fix $r, 0<r<1$, arbitrarily. Since

$$
\lim _{\delta \rightarrow 1} \iint_{\delta<|z|<1} f^{\#}(z)^{2} d x d y=0,
$$

it follows that, for each $\varepsilon>0$, there exists $\delta, 0<\delta<1$, such that

Since

$$
\iint_{\delta<|z|<1} f^{\#}(z)^{2} d x d y<\pi \varepsilon .
$$

$$
(r<)(r+\delta) /(1+\delta r)<|w|<1 \Rightarrow \Delta(w, r) \subset\{\delta<|z|<1\},
$$

it follows that

$$
\pi S\left(r, f_{w}\right)=\iint_{\Delta(w, r)} \int_{r} f^{\#}(z)^{2} d x d y<\pi \varepsilon,
$$

or $S\left(r, f_{w}\right)<\varepsilon$. By Lemma 3.2 (II), $f$ is a member of $\mathrm{N}_{0}$.

For the proof of $f \in \mathrm{UBC}$, we first note that

$$
\left(1-|z|^{2}\right) f_{w}^{\#}(z)=\left(1-\left|\varphi_{w}(z)\right|^{2}\right) f^{\#}\left(\varphi_{w}(z)\right) \leqq K
$$

for all $z$ and $w$ in $D$, because $f \in N$. Fix $R, 0<R<1$, and then let $R<r<1$. We have then

$$
T\left(r, f_{w}\right)=T\left(R, f_{w}\right)+\int_{R}^{r} t^{-1} S\left(t, f_{w}\right) d t \equiv \alpha+\beta .
$$

By (2.5) in Lemma 2.2,

$$
\pi \alpha=\iint_{|z|<R} f_{w}^{\#}(z)^{2} \log (R /|z|) d x d y \leqslant 2 \pi K^{2} \int_{0}^{R} \varrho\left(1-\varrho^{2}\right)^{-2} \log (R / \varrho) d \varrho \equiv C_{1}(R)<\infty .
$$

On the other hand, since

it follows that

$$
\pi t^{-1} S\left(t, f_{w}\right) \leqq R^{-1} A \quad \text { for } \quad R<t<r,
$$

$$
\pi \beta \leqq(1-R) R^{-1} A \equiv C_{2}(R)<\infty,
$$


which, together with (6.1), yields that

$$
\pi \sup _{w \in D} T\left(1, f_{w}\right) \leqq C_{1}(R)+C_{2}(R) .
$$

This completes the proof of Theorem 6.1.

Remark. There exists a holomorphic function $f$ in $D$ such that $f \notin \mathrm{N}$, yet

$$
\iint_{D}\left|f^{\prime}(z)\right|^{p} d x d y<\infty \text { for all } p, 0<p<2 ;
$$

see [9]. Therefore $f \notin U B C$, yet

$$
\iint_{D} f^{\#}(z)^{p} d x d y<\infty \text { for all } p, 0<p<2 .
$$

In other words, condition (6.2) for meromorphic $f$ does not necessarily assure that $f \in \mathrm{UBC}$.

\section{BMOA and VMOA}

Let $|J|$ be the linear Lebesgue measure of a subarc $J$ of the circle $\Gamma=\{|z|=1\}$. For each $f$ of complex $L^{1}(\Gamma)$ we set

$$
J(f)=(1 /|J|) \int_{J} f\left(e^{i t}\right) d t
$$

called the mean of $f$ on $J$. Then $f$ is said to have bounded mean oscillation on $\Gamma$, in notation, $f \in \operatorname{BMO}(\Gamma)$, if and only if the mean oscillation $J(|f-J(f)|)$ of $f$ on $J$, the mean of $|f-J(f)|$ on $J$, remains bounded as $J$ ranges over all subarcs of $\Gamma$. Furthermore, $f$ is said to have vanishing mean oscillation on $\Gamma$, in notation, $f \in \operatorname{VMO}(\Gamma)$, if and only if $f \in \operatorname{BMO}(\Gamma)$ and for each $\varepsilon>0$ there exists $\delta>0$ such that

$$
|J|<\delta \Rightarrow J(|f-J(f)|)<\varepsilon .
$$

For the properties of $\mathrm{BMO}(\Gamma)$ and $\operatorname{VMO}(\Gamma)$, see [6] and [8].

Let $H^{p}$ be the Hardy class consisting of $f$ holomorphic in $D$ such that $|f|^{p}$ has a harmonic majorant in $D$, where $0<p<\infty$. Each $f \in H^{p}$ has a boundary value $f\left(e^{i t}\right) \in C$, being the angular limit, at a.e. point $e^{i t} \in \Gamma$ and $f\left(e^{i t}\right)$ is of $L^{p}(\Gamma)$. For $f \in H^{p}$, the norm $\|f\|_{p} \geqq 0$ is defined by

$$
\|f\|_{p}^{p}=\left(|f|^{p}\right)^{\wedge}(0)=(1 / 2 \pi) \int_{0}^{2 \pi}\left|f\left(e^{i t}\right)\right|^{p} d t .
$$

By definition ([8, p. 90]; see also [2, Theorem 3.1, p. 34]),

$$
\begin{aligned}
& \mathrm{BMOA}=\left\{f \in H^{1} ; f\left(e^{i t}\right) \in \operatorname{BMO}(\Gamma)\right\}, \\
& \mathrm{VMOA}=\left\{f \in H^{1} ; f\left(e^{i t}\right) \in \operatorname{VMO}(\Gamma)\right\} .
\end{aligned}
$$

It is known (see [8, Theorem, p. 36]) that if $f \in \mathrm{BMOA}$, then for each $p, 1 \leqq p<\infty$,

$$
\sup _{w \in D}\left(|f-f(w)|^{p}\right)^{\wedge}(w)<\infty .
$$


An immediate consequence of (7.1) is that $f \in H^{p}$ for all $p$, because, for $p \geqq 1$,

$$
\left(|f|^{p}\right)^{\wedge} \leqq 2^{p-1}\left(|f-f(0)|^{p}\right)^{\wedge}+2^{p-1}|f(0)|^{p},
$$

where $\left(|f-f(0)|^{p}\right)^{\wedge}$ exists by (7.1), namely,

$$
\left(|f-f(0)|^{p}\right)^{\wedge}(0)<\infty .
$$

Conversely, if $f \in H^{1}$ and if (7.1) is valid for a certain $p, 1 \leqq p<\infty$, then $f \in \mathrm{BMOA}$.

Therefore, a holomorphic function $f$ in $D$ is of BMOA if and only if

$$
\sup _{w \in D}\left\|f_{w}-f(w)\right\|_{2}<\infty \text {. }
$$

Actually, setting $g=f-f(w)$ and considering Lemma 5.1, one calculates that

$$
\begin{gathered}
\left\|f_{w}-f(w)\right\|_{2}^{2}=\left(\left|g \circ \varphi_{w}\right|^{2}\right)^{\wedge}(0)=\left(|g|^{2} \circ \varphi_{w}\right)^{\wedge}(0) \\
=\left(|g|^{2}\right)^{\wedge} \circ \varphi_{w}(0)=\left(|g|^{2}\right)^{\wedge}(w)=\left(|f-f(w)|^{2}\right)^{\wedge}(w) .
\end{gathered}
$$

A straightforward modification of the proof of $[8$, Theorem, p. 36] yields the VMOA version:

If $f \in \mathrm{VMOA}$, then for each $p, 1 \leqq p<\infty$,

$$
\lim _{|w| \rightarrow 1}\left(|f-f(w)|^{p}\right)^{\wedge}(w)=0 .
$$

Conversely, if $f \in \mathrm{BMOA}$ and (7.4) for a certain $p, 1 \leqq p<\infty$, holds, then $f \in \mathrm{VMOA}$.

However, it must be emphasized that the condition $f \in \mathrm{BMOA}$ in the preceding sentence can be dropped. Namely, if a holomorphic $f$ in $D$ satisfies (7.4) for a $p$, $1 \leqq p<\infty$, then $f \in \mathrm{VMOA}$. To ascertain this it suffices to show that $f \in \mathrm{BMOA}$ under the condition (7.4). First, there exists $\delta, 0<\delta<1$, such that

$$
\delta<|w|<1 \Rightarrow\left(|f-f(w)|^{p}\right)^{\wedge}(w)<1 .
$$

On replacing 0 in (7.2) by $r_{0}=(1+\delta) / 2$, we observe that $f \in H^{p}$. Now, for $w$, $|w| \leqq r_{0}$,

$$
\left(|f-f(w)|^{p}\right)^{\wedge}(w) \leqq 2^{p-1}\left(|f|^{p}\right)^{\wedge}(w)+2^{p-1}|f(w)|^{p} .
$$

The right-hand side is apparently bounded for $|w| \leqq r_{0}$, which, together with (7.5), shows that (7.1) is valid. Consequently, $f \in \mathrm{BMOA}$.

By the observation in the preceding paragraph we can now conclude that a holomorphic function $f$ in $D$ is of VMOA if and only if

$$
\lim _{|w| \rightarrow 1}\left\|f_{w}-f(w)\right\|_{2}=0
$$

a VMOA counterpart of (7.3).

We propose

Theorem 7.1. The inclusion formulae

hold.

$$
\mathrm{BMOA} \subset \mathrm{UBC} \text { and } \mathrm{VMOA} \subset \mathrm{UBC}_{0}
$$


For the proof we first consider the holomorphic analogue $T^{*}(r, f)$ of the Shimizu-Ahlfors characteristic function basing on the identity

$$
\Delta\left(|f|^{2}\right)=4\left|f^{\prime}\right|^{2}
$$

for $f$ holomorphic in $D$ instead of $\Delta \log \left(1+|f|^{2}\right)=4 f^{\# 2}$.

For $f$ holomorphic in $D$ we set

$$
M(r, f)=\left[(1 / 2 \pi) \int_{0}^{2 \pi}\left|f\left(r e^{i t}\right)\right|^{2} d t\right]^{1 / 2}, \quad 0<r \leqq 1,
$$

where $M(1, f)=\lim _{r \rightarrow 1} M(r, f)$. If $f \in H^{2}$, then $\|f\|_{2}=M(1, f)$. Since (7.7) holds, the Green formula yields

where

$$
r(d / d r)\left[M(r, f)^{2}\right]=A(r, f)
$$

$$
A(r, f)=(2 / \pi) \iint_{|z|<r}\left|f^{\prime}(z)\right|^{2} d x d y
$$

is the holomorphic analogue of $S(r, f)$. Setting

$$
T^{*}(r, f)=\int_{0}^{r} t^{-1} A(t, f) d t, \quad 0<r \leqq 1,
$$

one obtains the formula

$$
M(r, f)^{2}-|f(0)|^{2}=T^{*}(r, f), \quad 0<r \leqq 1 .
$$

Applying (7.8) to $g=f_{w}-f(w)(g(0)=0)$, one observes from (7.3) and (7.6), together with

that

$$
T^{*}(r, g)=T^{*}\left(r, f_{w}\right)
$$

$f \in \mathrm{BMOA}$ if and only if $\sup _{w \in D} T^{*}\left(1, f_{w}\right)<\infty$,

while

Since

$$
f \in \mathrm{VMOA} \text { if and only if } \lim _{|w| \rightarrow 1} T^{*}\left(1, f_{w}\right)=0 \text {. }
$$

$$
T^{*}(r, f)=(2 / \pi) \iint_{|z|<r}\left|f^{\prime}(z)\right|^{2} \log (r /|z|) d x d y
$$

for $f$ holomorphic in $D$ and for $0<r \leqq 1$, the analogue of (2.5) holds, and it is now an easy exercise to obtain the following holomorphic counterpart of Theorem 2.2.

Lemma 7.1. Let $f$ be holomorphic in $D$. Then the following propositions hold. (I) $f \in \mathrm{BMOA}$ if and only if

$$
\sup _{w \in D} \int_{D} \int\left|f^{\prime}(z)\right|^{2} G(z, w) d x d y<\infty .
$$


(II) $f \in \mathrm{VMOA}$ if and only if

$$
\lim _{|w| \rightarrow 1} \iint\left|f_{D}^{\prime}(z)\right|^{2} G(z, w) d x d y=0
$$

Lemma 7.1 (I) is known [6, Proposition 7.2.13, p. 85]. Theorem 7.1 now follows from Theorem 2.2 and Lemma 7.1, because $\left|f^{\prime}\right| \geqq f^{\#}$ for $f$ holomorphic in $D$.

Remark. At this point we remark that if $f$ is holomorphic in $D$ and if

$$
\iint_{D}\left|f^{\prime}(z)\right|^{2} d x d y<\infty
$$

then $f \in \mathrm{VMOA}$. By the theorem at the bottom of $[8, \mathrm{p} .50]$ it suffices to show that

$$
\lim _{|J| \rightarrow 0} \mu_{f}(R(J)) /|J|=0
$$

where $|J|<\pi$, and $R(J)$ is the annular trapezoid

and

$$
\{z \in D ; z /|z| \in J, 1-|z| \leqq|J| /(2 \pi)\}
$$

$$
\mu_{f}(R(J))=\iint_{R(J)}(1-|z|)\left|f^{\prime}(z)\right|^{2} d x d y
$$

Since $1-|z| \leqq|J|(2 \pi)$, it follows that

$$
\mu_{f}(R(J)) \leqq[|J| /(2 \pi)] \iint_{R(J)}\left|f^{\prime}(z)\right|^{2} d x d y \leqq[|J| /(2 \pi)] \iint_{1-|J| /(2 \pi)<|z|<1}\left|f^{\prime}(z)\right|^{2} d x d y .
$$

Therefore $\mu_{f}(R(J)) /|J| \rightarrow 0$ as $|J| \rightarrow 0$.

A natural question then arises: Can the conclusion in Theorem 6.1 be replaced by $f \in \mathrm{UBC}_{0}$ ?

\section{References}

[1] Dufresnoy, J.: Sur les domaines couverts par les valeurs d'une fonction méromorphe ou algébroïde. - Ann. École Norm. Sup. (3) 58, 1941, 179--259.

[2] Duren, P. L.: Theory of $H^{p}$ spaces. - Academic Press, New York-London, 1970.

[3] Hayman, W. K.: Meromorphic functions. - Clarendon Press, Oxford, 1964.

[4] John, F., and L. Nirenberg: On functions of bounded mean oscillation. - Comm. Pure Appl. Math. 14, 1961, 415-426.

[5] Lehto, O., and K. I. VirTANen: Boundary behaviour and normal meromorphic functions. Acta Math., 97, 1957, 47-65.

[6] Petersen, K. E.: Brownian motion, Hardy spaces and bounded mean oscillation. - Cambridge University Press, Cambridge-London-New York-Melbourne, 1977. 
[7] Sarason, D.: Functions of vanishing mean oscillation. - Trans. Amer. Math. Soc. 207, 1975, 391-405.

[8] Sarason, D.: Function theory on the unit circle. - Virginia Polytechnic Institute and State University, Blacksburg, 1978.

[9] YamashitA, S.: A non-normal function whose derivative has finite area integral of order $0<p<2$. - Ann. Acad. Sci. Fenn. Ser. A I Math. 4, 1978/1979, 293-298.

[10] YamashitA, S.: Gap series and $\alpha$-Bloch functions. - Yokohama Math. J. 28, 1980, 31-36.

[11] Yamashita, S.: Non-normal Dirichlet quotients and non-normal Blaschke quotients. Proc. Amer. Math. Soc. 80, 1980, 604-606.

[12] Yamashita, S.: Criteria for functions to be Bloch. - Bull. Austral. Math. Soc. 21, 1980, $223-227$.

[13] Yamashita, S.: Bi-Fatou points of a Blaschke quotient. - Math. Z. 176, 1981, 375-377.

Tokyo Metropolitan University

Department of Mathematics

Fukasawa, Setagaya

Tokyo 158

Japan

Received 1 June 1982 